\title{
High performance mixed optical CDMA system using ZCC code and multiband OFDM
}

\author{
Nawawi N. M. ${ }^{1,{ }^{*}}$, Anuar M. S. ${ }^{1}$, Junita $M . N^{1}$ and Rashidi C. B.M. ${ }^{1}$ \\ ${ }^{1}$ Optical Research Group, Advanced Communication Engineering Centre of Excellence (ACE-COE), School of Computer \& \\ Communication Engineering, Universiti Malaysia Perlis (UNIMAP), 02600 Perlis, Malaysia.
}

\begin{abstract}
In this paper, we have proposed a high performance network design, which is based on mixed optical Code Division Multiple Access (CDMA) system using Zero Cross Correlation (ZCC) code and multiband Orthogonal Frequency Division Multiplexing (OFDM) called catenated OFDM. In addition, we also investigate the related changing parameters such as; effective power, number of user, number of band, code length and code weight. Then we theoretically analyzed the system performance comprehensively while considering up to five OFDM bands. The feasibility of the proposed system architecture is verified via the numerical analysis. The research results demonstrated that our developed modulation solution can significantly enhanced the total number of user; improving up to $80 \%$ for five catenated bands compared to traditional optical CDMA system, with the code length equals to 80 , transmitted at $622 \mathrm{Mbps}$. It is also demonstrated that the BER performance strongly depends on number of weight, especially with less number of users. As the number of weight increases, the BER performance is better.
\end{abstract}

\section{Introduction}

In the past two decades, optical CDMA spaces have been studied extensively. An OCDMA attracts considerable attention due to asynchronous access capability [1] and provides security in transmission network as its distinguished each channel by a specific optical code [2]. Many research on finding the most suitable form of optical CDMA coding system has been widely investigated for coherent and incoherent optical multiple access techniques [3][4][5]. Recently, there are a number of published studies describing the hybrid technology by combining OCDMA system with different technologies to take benefits of both technologies. Such systems are wavelength division multiplexing (WDM) [6][7][8], subcarrier multiplexing (SCM) [9] and OFDM[10]. The application of the advanced modulation technique in the higher speed optical CDMA transmission system has become a new research hotspot.

OFDM modulation technique has aroused extensive research interests due to its resistance to inter symbol interference (ISI) caused by a dispersive channel [11]. It is a well-known developing technology for high data rates

\footnotetext{
* Corresponding author: norizan@unimap.edu.my
}

system. This tool spreads the data to be transmitted in the medium over huge number of subcarriers, therefore can efficiently use the allocated bandwidth. Compared with the conventional modulation mode on-off keying, OFDM has the following advantages: high spectral efficiency, dynamic bandwidth allocation and adaptive bit rate functionalities, and high tolerance to multi-path interference, channel dispersion and frequency-selective fading [1][12][13].

Motivated by the advance contribution in modulation technologies, this project aims to explore and embed a new modulation scheme into optical domain based on multiband QAM-OFDM. This technique is then combined with OCDMA system to achieved large bandwidth efficiencies while maintaining the high data rates system. Moreover, the maximum channel capacity can be enhanced by sending multiple OFDM bands and/or increasing OCDMA codewords. Comparison between conventional OCDMA with multiband/catenated-OFDMOCDMA is provided.

The rest of this paper is structured as follows. Section 2 describes the system architecture of an OCDMA transmission network based on ZCC to transmit catenated 
band of OFDM signals. End of this section discuss the theoretical model for performance analysis. Results and discussion is provided in Section 3. Finally, some conclusions are drawn in Section 4.

\section{Operation principle}

Fig. 1 schematically depicts our proposed design system that exploits OCDMA-ZCC and catenated OFDM technologies. The developed system employs $n$-bands OFDM signals with ZCC codeword simultaneously over optical channel. As can be seen in the figure, each input data streams are passed through OFDM modulators to generate corresponding OFDM signals. Each subcarrier of OFDM signal encoded with 16-QAM symbol and generated at a center frequency $f_{l}, . ., f_{n}$, respectively. OFDM modulator which then modulates a digital signal into multiple orthogonal subcarriers.

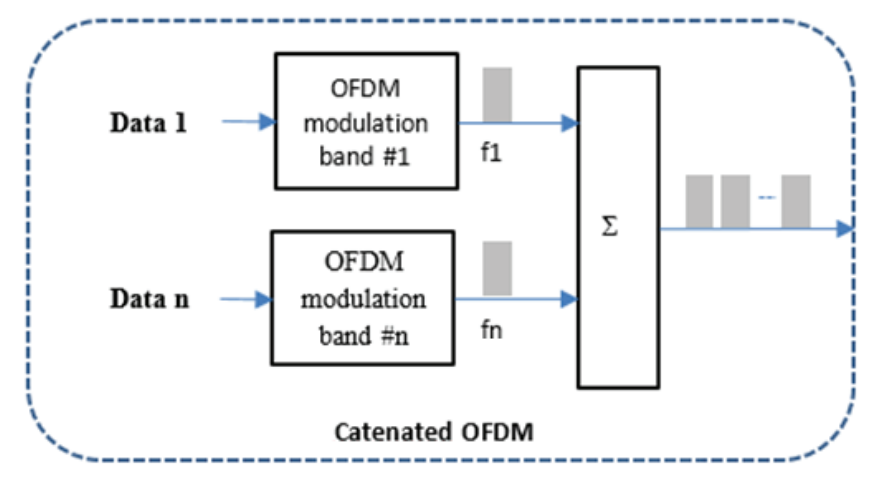

Fig. 1 Multiband catenated OFDM

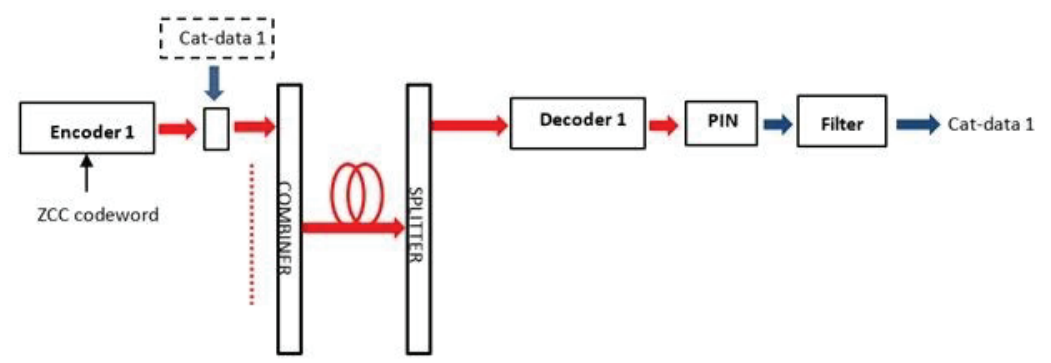

Fig. 2 Catenated-OFDM Optical CDMA based on ZCC architecture 
Next, the sum of the OFDM signal bands then optically modulated onto sequence through external Mach Zehnder modulator (MZM), each code sequence is assigned with different ZCC code structure as shown in Fig. 2. The $n$th OFDM band at $k$ subcarriers according to [14] is expressed as

$$
s(t)_{c a t}=\sum_{k=1}^{N-1} X_{n}[k] \cdot e^{\left(j 2 \pi f_{k} t\right)} \sum_{n=0}^{N_{s b}} m_{n} \cdot e^{j 2 \pi n f_{m} t}
$$

where $f_{k}$ and $f_{m}$ are the frequencies of $k$ th subcarrier and $m$ th band frequencies respectively, $X_{n}[k]$ denotes the OFDM information symbol.

Then the modulated code sequences are combined together via an optical combiner and transmitted through the optical fiber. At the receiver, an optical splitter is used to split the modulated code sequences to various paths according to the number of receivers. Only the matched code sequences are decoded while the unmatched code sequences will be filtered out. Another advantage using the proposed catenated OFDM scheme is the overall spectrum can be readily partitioned with electrical filter at the receiver to separate each band. Each channel that brings mutltiband OFDM signals is assigned a particular code sequence.

The signal to noise ratio (SNR) and BER for combining catenated OFDM signals with ZCC OCDMA structure is given by

$$
S N R=\frac{\left(R \cdot \frac{P_{r} w}{L} \cdot \sum_{k=0}^{N_{s c}-1} x_{k} e^{j 2 \pi f_{k} t} \cdot \sum_{n=1}^{N_{s k}} m_{n} e^{j 2 \pi n f_{m} t}\right)^{2}}{2 e\left(R \cdot \frac{P_{r} w}{L} \cdot \sum_{k=0}^{N_{s c}-1} x_{k} e^{j 2 \pi f_{k} t} \cdot \sum_{n=1}^{N_{s b}} m_{n} e^{j 2 \pi n f_{m} t}\right) B+\frac{4 k T B}{R_{L}}}
$$

where $L$ and $w$ are ZCC code length and weight respectively. ZCC code properties were calculated based on equation in [15].

$$
B E R=\frac{\sqrt{M}-1}{\sqrt{M} \log _{2} \sqrt{M}} \operatorname{erfc} \sqrt{\frac{3 \log _{2} M}{2(M-1)} S N R}
$$

\section{Result and Discussion}

In this section, we evaluate the performance, in terms of bit error rate, of the proposed modulation scheme using multiband OFDM and OCDMA-ZCC. The numerical results are obtained based on the expressions derived in Section 2. The BER is evaluated with different number of parameters consisting of the effective power, number of users and code length. The transmission of catenatedOFDM signal, whose parameters according to Table 1 is analyzed.
Table 1. Parameters for numerical analysis

\begin{tabular}{lc}
\hline PARAMETER & VALUE \\
\hline Number of subcarrier & 256 \\
Number of band & 1 to 5 bands \\
Modulation Mapping, $M$ & $16-\mathrm{QAM}$ \\
Data rate & $622 \mathrm{Mbps}$ \\
Number of bands & $1-5$ \\
Number of samples & 65536 \\
Responsivity, $R$ & 0.7 \\
Bandwidth & $311 \mathrm{MHz}$ \\
Load Resistor, $R_{L}$ & 1030 \\
Electron charge, $e$ & $1.6 \times 10^{-19} \mathrm{C}$ \\
Boltzmann's constant, $k$ & $1.38 \times 10^{-23} \mathrm{~J} / \mathrm{K}$ \\
Absolute temperature, $T$ & $300 \mathrm{~K}$ \\
\hline
\end{tabular}

Firstly, in Fig. 3, the BER of the proposed scheme and conventional OCDMA based on ZCC code are demonstrated versus the number of permissible users with different value of effective power. It is seen that the use of new scheme helps to significantly increase the number of users compared to original OCDMA-ZCC system. More specifically, for BER less than $10 \mathrm{E}^{-9}$, the catenatedOFDM/OCDMA-ZCC system with 5 bands achieved more than 200 number of users while only 110 number of user is allowed for case of OCDMA-ZCC with $P_{r}=$ $10 \mathrm{dBm}$. BER performance degrades with respects to lower effective power. Hence, it is worth noting that, for proposed scheme at larger OFDM bands (5 bands), the BER performance is far better than traditional OCDMA$\mathrm{ZCC}$ at same effective power level. By transmitting 5 bands simultaneously, the available electrical bandwidth is fully utilized and increase the spectral efficiency of the system.

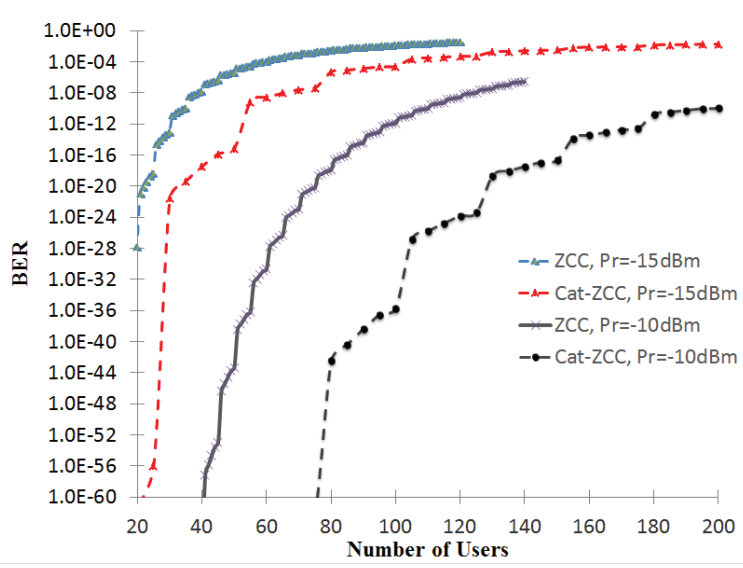

Fig. 3. Comparison of number of users for catenatedOFDM/OCDMA-ZCC with conventional OCDMA-ZCC

Secondly, to scrutinize the effect of code length on number of user performance, the model has been run for different code length in range of 10 to 80 and compared with traditional OCDMA-ZCC system. As shown in Fig. 4 , the number of user dramatically increases with the 
increased code length for catenated-OFDM at 5 bands. The number of user improves approximately $80 \%$ between catenated-OFDM and conventional ZCC for a given code length of 80 . Therefore, the catenated-ZCC technique shows better performance for larger OFDM bands at same bandwidth allocation.

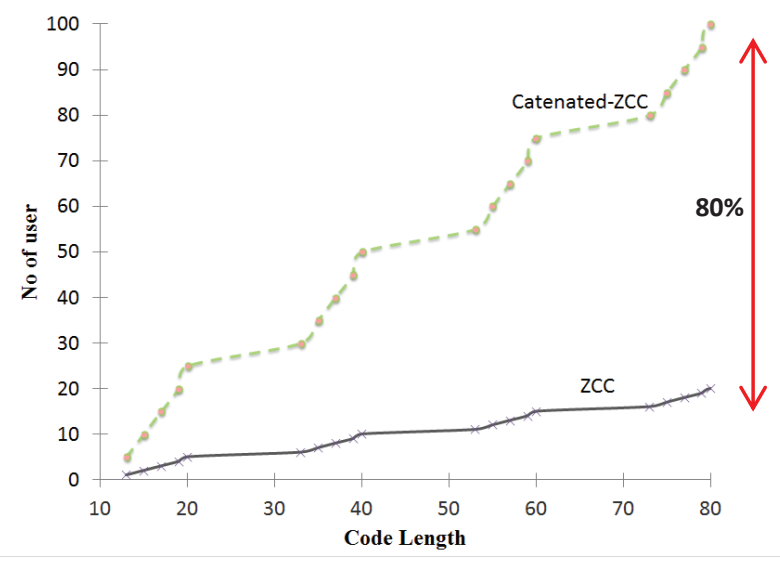

Fig. 4. Comparison of code length for catenatedOFDM/OCDMA-ZCC with conventional OCDMA-ZCC

Finally, Fig. 5 quantifies the effect number of users for different number of weight. Previous results are based on number of weight, $w=4$. Here, we consider four value of weight; $\mathrm{w}=2, \mathrm{w}=4, \mathrm{w}=6$ and $\mathrm{w}=8$. It is demonstrated that the BER performance strongly depends on number of weight, especially with less number of users. As the number of weight increases, the BER performance is better. This is due to more power density will be added to the overall spectrum while increasing the number of weight. However, when number of weight larger, bigger bandwidth is required and hence, increase the cost of the system.

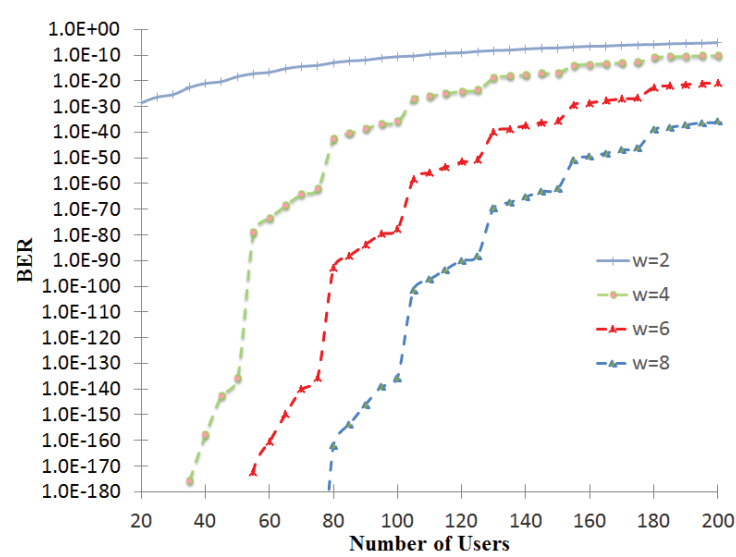

Fig. 5. BER versus number of users

\section{Conclusion}

In this paper, we have proposed high spectral efficiency modulation technique employing multiband catenated-OFDM technique and ZCC OCDMA system. The developed system is able to provide better BER performance at their respective parameters, higher permitted number of users and high spectral efficiency. To the best of our knowledge, it is the first time the performance of OCDMA system is numerically analyzed using multiband OFDM signals. We have also comprehensively investigated the impacts of number of OFDM bands, on the performance. The feasibility of our proposed scheme is verified by numerical evaluation and proves that overall system capacity enhancement approximately $80 \%$ with code length of 80 . The numerical results also show that inter band interferences is main factor that effect on performance of the proposed systems in sending more bands simultaneously.

\section{References}

1. X. Wang, 7th IEEE Int. Conf. Optoelectron. Fiber Opt. Photonic, 2004, pp. 1-6 (2004)

2. M. S. Anuar, S. A. Aljunid, A. R. Arief, M. N. Junita, and N. M. Saad, Opt. - Int. J. Light Electron Opt., 124, no. 4, pp. 371-375 (2013)

3. T. Hussein, S. A. Aljunid, H. Adnan, R. A. Ahmad, and N. M. Saad, Opt. Fiber Technol., 17, no. 4, pp. 273-280 (2011)

4. M. S. Anuar, S. A. Aljunid, N. M. Saad, and S. M. Hamzah, Opt. Commun., 282, no. 14, pp. 26592664 (2009)

5. N. D. Keraf, S. A. Aljunid, A. R. Arief, and P. Ehkan, Advanced Computer and Communication Engineering Technology, pp. 129-140 (2014)

6. I. A. M. Ashour, S. Shaari, and P. Susthitha Menon and Hesham A . Bakarman, Photonic Technology Laboratory, 8, no. 10, pp. 1718-1729 (2012)

7. F. Hou and M. Yang, Opt. - Int. J. Light Electron Opt., 125, no. 15, pp. 4150-4153 (2014)

8. N. Ahmed, M. A. Rashid, M. Islam, 26 th International Telecommunication Networks and Applications Conference (ITNAC), (2016)

9. M. N. Junita, S. A. Aljunid, Thanaa Hussein Abd, M. S. Anuar, A. R. Arief, R. A. Rahim, $20112^{\text {nd }}$ International Conference on Photonics (2011)

10. A. O. Aldhaibani, S. A. Aljunid, M. S. Anuar, and A. R. Arief, J. Theor. Appl. Inf. Technol., 66, no. 2, pp. 634-637 (2014)

11. M. Singh and K. Kaur, Ijircce, 2, no. 12, pp. 47934797 (2013)

12. I. B. Djordjevic and B. Vasic, Opt. Express, 14, 
no. 9, pp. 3767-3775 (2006)

13. Y. Yin, W. Lan, T. Lin, C. Wang, and M. Feng, 35, no. 2, pp. 258-264 (2017)

14. A. I. Technology, N. M. Nawawi, M. N. Junita, A. K. Rahman, S. A. Aljunid, and C. Engineering, 91, no. 1 (2016)

15. M. S. Anuar, S. A Aljunid, A. R. Arief, and N. M. Saad, 7th Int. Symp. High-Capacity Opt. Networks Enabling Technol. HONET 2010, pp. 128-132 (2010) 